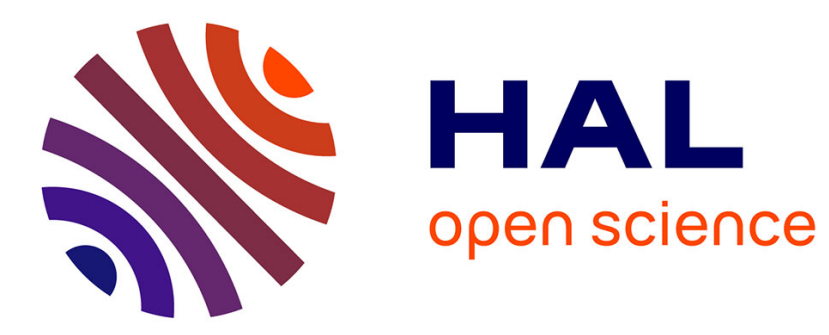

\title{
Origine et structure du champ magnétique permanent dans la magnétosphère et l'espace interplanétaire Jean-François Crifo
}

\section{To cite this version:}

Jean-François Crifo. Origine et structure du champ magnétique permanent dans la magnétosphère et l'espace interplanétaire. Revue de Physique Appliquée, 1970, 5 (1), pp.195-207. 10.1051/rphysap:0197000501019500 . jpa-00243360

\section{HAL Id: jpa-00243360 https://hal.science/jpa-00243360}

Submitted on 1 Jan 1970

HAL is a multi-disciplinary open access archive for the deposit and dissemination of scientific research documents, whether they are published or not. The documents may come from teaching and research institutions in France or abroad, or from public or private research centers.
L'archive ouverte pluridisciplinaire HAL, est destinée au dépôt et à la diffusion de documents scientifiques de niveau recherche, publiés ou non, émanant des établissements d'enseignement et de recherche français ou étrangers, des laboratoires publics ou privés. 


\title{
ORIGINE ET STRUGTURE DU GHAMP MAGNÉTIQUE PERMANENT DANS LA MAGNÉTOSPHÈRE ET L'ESPAGE INTERPLANÉTAIRE
}

\author{
Par J.-F. GRIFO, \\ Centre National de la Recherche Scientifique (G.N.R.S.), Laboratoire de Physique Stellaire et Planétaire,

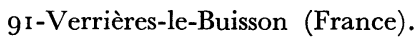

\begin{abstract}
Résumé. - La structure du champ magnétique dans l'espace interplanétaire est déterminée par l'expansion de la couronne solaire (vent solaire) ainsi que par l'état instantané du champ magnétique de la photosphère; le présent exposé commence donc par une revue rapide des connaissances actuelles concernant le vent solaire. La seconde partie est consacrée au champ magnétosphérique. On expose à cette occasion le problème de l'interaction entre ce champ et le vent solaire, interaction qui détermine la forme de la cavité magnétosphérique et la configuration du champ dans les régions externes de la cavité. En conclusion, un aperçu est donné sur l'interaction du vent solaire avec la Lune.
\end{abstract}

Introduction. - On connaît actuellement dans le système solaire trois objets fortement magnétisés : le Soleil lui-même, la Terre et la planète Jupiter. En ce qui concerne cette dernière planète, l'existence d'un champ intrinsèque a été établie par les radioastronomes: l'émission intense observée en ondes décimétriques peut s'expliquer par le rayonnement synchroton d'électrons relativistes $(10$ à $100 \mathrm{MeV})$ piégés dans un champ de 1 à 10 gauss, ce qui correspond à un moment magnétique $M_{\longleftarrow} \approx 5 \times 10^{4} M_{\oplus}$; rappelons que le moment magnétique terrestre est [1] $M_{\oplus}=7,9$ $\times 10^{22} \mathrm{~A} \cdot \mathrm{m}^{2}$. Nous reviendrons plus loin sur le champ magnétique solaire, dont la mesure au niveau de la photosphère est déduite de l'effet Zeeman optique [2].

Quant aux autres planètes, proches ou lointaines, on ne peut actuellement que placer des limites supérieures sur leur moment intrinsèque, que ceci résulte de mesures magnétiques sur sondes spatiales (Lune [3], Vénus [4], Mars [5]) ou de méthodes radioélectriques (Saturne [6]). Ainsi :

$$
M_{3}<10^{-6} M_{\oplus}, \quad M_{\uparrow}<10^{-2} M_{\oplus}
$$

et

$$
M{ }^{\star}<3 \times 10^{-4} M_{\oplus} .
$$

Bien que cela puisse surprendre, ce sont les régions externes du Soleil (photosphère et couronne) qui déterminent la structure du champ magnétique dans l'espace interplanétaire, y compris au voisinage de la Terre et de Jupiter; ceci résulte du fait que la couronne solaire n'est pas en état d'équilibre statique, mais d'expansion continue. Le gaz ionisé qu'elle émet (vent solaire) « emporte » avec lui un champ magnétique ambiant; il interagit de plus avec le champ magnétique des planètes magnétisées, ou avec la surface elle-même des autres planètes; il en résulte en général un « sillage » qui s'étend sur les distances de plusieurs centaines de fois le rayon de la planète dans la direction antisolaire.

Nous allons décrire en premier lieu les propriétés actuellement connues du vent solaire, puis nous aborderons le problème des interactions avec les planètes, en nous limitant pratiquement au cas de la Terre.
I. Le vent solaíre et le champ magnétique interplanétaire. - Nous ferons usage dans ce qui suit de coordonnées sphériques $(r, \theta, \varphi)$ ayant pour origine le centre du Soleil.

I.1. ÉQUILIBRE DE LA GOURONNE SOLAIRE. - a) Équations fondamentales. - Nous commencerons par oublier que la couronne est un milieu ionisé, c'est-à-dire que nous allons utiliser les équations d'équilibre des gaz neutres.

L'équilibre hydrostatique d'une atmosphère gazeuse placée dans un champ de pesanteur $g$ dirigé suivant $r$ s'écrit, $p$ étant la pression du gaz, $n$ sa densité et $m$ sa masse moléculaire moyenne :

$$
\mathrm{d} p=-n m g \mathrm{~d} r .
$$

Soit, en introduisant la température $T$ et en explicitant le champ de pesanteur en fonction de la masse du Soleil $M_{\odot}$ :

$$
\frac{\mathrm{d}}{\mathrm{d} r}(2 n k T)+\frac{G M_{\odot} n m}{r^{2}}=0 .
$$

En intégrant entre un niveau de référence $r_{0}$ et $r$, on obtient :

$$
n(r) T(r)=n_{0} T_{0} \exp \left\{-\frac{G M_{\odot} m}{2 k} \int_{r_{0}}^{r} \frac{\mathrm{d} r}{r^{2} T(r)}\right\} .
$$

La fonction $T(r)$ joue le rôle d'une équation d'état du gaz, déterminée par le bilan énergétique (inconnu) de celui-ci. Il est clair en effet que le chauffage du gaz contribue à déterminer son équilibre dans le champ de gravitation. On voit que si $T(r)$ tend vers 0 à l' $\infty$ moins vite que $1 / r$, la pression $k n(r) T(r)$ ne tend pas vers zéro : preuve que l'équation (1) ne convient pas; en d'autres termes, l'atmosphère n'est pas en équilibre hydrostatique. C'est effectivement le cas de la couronne solaire qui est à peu près isotherme. L'hypothèse la plus simple est alors celle d'un écoulement stationnaire : une tranche $\mathrm{d} r$ de gaz en expansion accroît sa vitesse de :

$$
(n m \mathrm{~d} r) \frac{\mathrm{d} v}{\mathrm{~d} t}=-\mathrm{d}(2 n k T)-n m g \mathrm{~d} r .
$$


La loi de conservation de la masse s'écrit, dans le cas d'une atmosphère sphérique :

$$
n v r^{2}=\text { Cte. }
$$

En différenciant (3), combinant avec (2), on obtient :

$\frac{1}{v} \frac{\mathrm{d} v}{\mathrm{~d} r}\left(\frac{v^{2}}{C^{2}}-1\right)=\frac{1}{r}\left(2-\frac{g r}{C^{2}}-\frac{r}{C^{2}} \cdot \frac{\mathrm{d}\left(C^{2}\right)}{\mathrm{d} r}\right)$

où :

$$
C^{2}=2 k T / m
$$

est la vitesse thermique moyenne.

On se reportera à [7] pour une discussion générale de cette équation. Dans l'hypothèse simple où la couronne est supposée isotherme, le dernier terme du second membre de (4) disparaît, et on obtient aisément :

$$
\frac{v^{2}}{C_{0}^{2}}-\log \frac{v^{2}}{C_{0}^{2}}=4 \log \frac{r}{r_{\mathrm{C}}}+4 \frac{r_{\mathrm{C}}}{r}-3
$$

où $r_{\mathrm{C}}$ est l'altitude critique solution de :

$$
r_{\mathrm{C}}=\frac{2\left[C_{0}\left(r_{\mathrm{C}}\right)\right]^{2}}{G M_{\odot}} .
$$

La figure 1 représente $v(r)$ solution de (6) pour plusieurs températures coronales. L'équation (3) permet d'en déduire la densité $n(r)$.

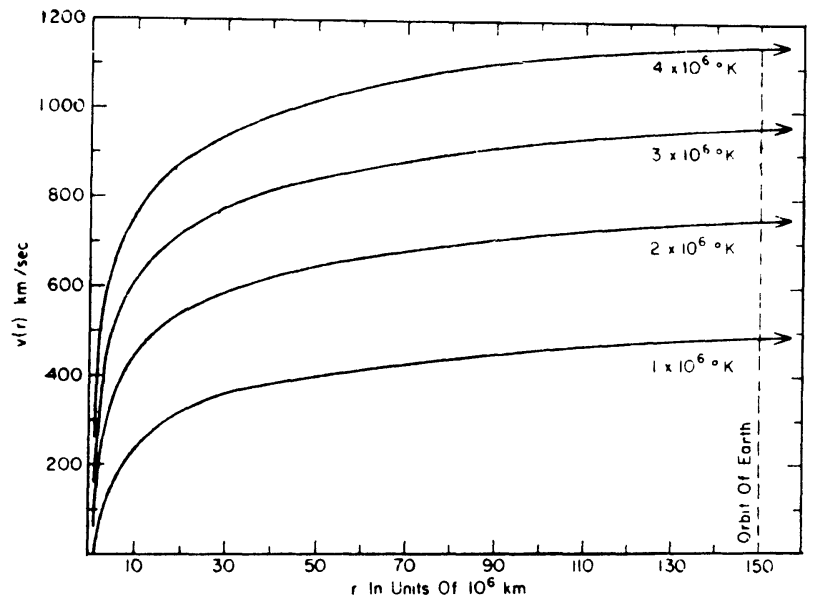

Fig. 1. - Loi de variation de la vitesse d'expansion de la couronne en fonction de la distance, pour plusieurs températures de la couronne supposée isotherme [7].

Avant d'examiner les résultats expérimentaux, soulignons que la théorie ci-dessus, due à Parker [7], suppose :

1) Que la couronne se comporte comme un fluide, et que

2) Un apport d'énergie maintient une température coronale élevée.

Enfin, on peut se demander jusqu'à quelle distance (4) a un sens. Il est raisonnable de penser que (4) n'a plus de sens lorsque la pression cinétique $n m v^{2}$ du vent est égale à la pression interstellaire $P_{i}$; en prenant pour celle-ci la pression $B_{i}^{2} / 2 \mu_{0}$ du champ magnétique interstellaire, on trouve [8]:

$r_{\max } \approx 60$ unités astronomiques $\left({ }^{1}\right)$.

(1) Unité astronomique $=$ distance Terre-Soleil $=150$ $\times 10^{6} \mathrm{~km}$.
La région balayée par le vent solaire, ou « héliosphère ", s'étend donc au-delà de la plus éloignée des planètes, Pluton (40 U.A.). On voit que pour longtemps encore les mesures magnétiques directes auront lieu à l'intérieur de l'héliosphère.

b) Mesures directes du vent solaire. - Depuis les premiers résultats de Mariner II en 1962, une grande masse de résultats se sont accumulés, concernant tant les propriétés globales que la structure fine du vent (fonctions de distribution, inhomogénéités, variations temporelles, ondes, etc.). On se reportera par exemple à [9] qui récapitule l'acquis à la fin de l'année 1968.

Ces mesures sont évidemment concentrées au voisinage de l'orbite terrestre, avec quelques exceptions fournies par les sondes Vénus, Mars et Pioneer.

Les chiffres moyens sont les suivants, à proximité de l'orbite terrestre :

- Vitesse d'expansion : $320 \mathrm{~km} / \mathrm{s}$;

- Densité de protons : $5 \mathrm{~cm}^{-3}$;

- Composition $\mathrm{He}^{++}: 5 \%$;

- Direction d'expansion : contenue dans le plan de l'écliptique, présentant un écart de $1,5^{\circ}$ par rapport à la direction du Soleil;

- Température protonique $4 \times 10^{4} \mathrm{oK}$ (anisotrope); - Température électronique $10^{8} \mathrm{oK}$ (anisotrope).

Cependant, comme nous le montrerons plus loin, toutes ces grandeurs varient considérablement dans le temps, en particulier par suite de la rotation solaire (période de 27 jours).

I.2. LE GHAMP MAGNÉTIQUE DANS LE VENT SOLAIRE. - a) Structure du champ. - Dans ce qui précède, on a traité la couronne solaire comme un gaz neutre. Comme nous nous intéressons ici au champ magnétique, nous allons être obligés de renoncer à cette simplification.

Il existe, à la base de la couronne, un champ magnétique (photosphérique) [2]. La figure 2 montre une

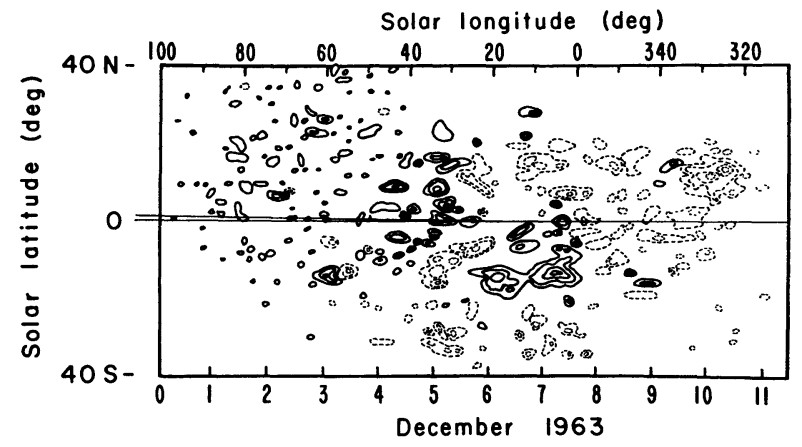

FIg. 2. - Un exemple de répartition du champ magnétique photosphérique. Les contours en traits pleins correspondent à un champ dirigé vers l'extérieur du Soleil; les contours pointillés à un champ dirigé vers l'intérieur. Ces contours correspondent aux intensités 2 , $4,8,12$ et 25 gauss [10].

carte de ce champ; on voit que la valeur moyenne est de l'ordre de quelques gauss, et qu'il existe une série de régions de polarités opposées, où le champ est plus intense. (N.-B. - Ces régions ne sont pas des taches solaires, lesquelles n'apparaissent pas sur la figure.) Introduisons le rapport :

$$
\beta=\frac{n m v^{2}+3 n k T}{\left(B^{2} / 2 \mu_{0}\right)} .
$$


Si $\beta<1$, le champ magnétique détermine le mouvement du gaz ionisé, si $\beta>1$, le gaz ionisé détermine la structure du champ; nous avons donc implicitement supposé $\beta>1$ dans le traitement du $\S$ I.1.

Avant d'évaluer le rapport $\beta$, nous allons rechercher la structure du champ en supposant $\beta>1$.

Plaçons-nous dans un référentiel solaire : à basse altitude, l'expansion du fluide a lieu verticalement, car l'étude du champ magnétique photosphérique montre que celui-ci est pratiquement radial. Autrement dit, dans un référentiel absolu, on a les composantes de vitesse :

$$
\begin{gathered}
v_{\varphi}=\omega_{\odot} R_{\odot} \\
v_{r}=v(r) .
\end{gathered}
$$

Or la figure 1 montre que $v_{r} \rightarrow V \gg v\left(R_{\odot}\right)$, tandis que $v_{\varphi}$ reste inchangée (aucun mécanisme plausible ne peut transférer une composante de vitesse capable d'assurer la corotation du gaz, lorsque la densité devient très faible).

On a donc, toujours dans un référentiel absolu :

$$
\begin{aligned}
& \dot{\varphi}=\omega_{\odot}\left(R_{\odot} / r\right) \approx 0 \text { dès que } r \gg R_{\odot}{ }^{(2)} \\
& \dot{r}=v(r) .
\end{aligned}
$$

Considérons alors un point $\mathrm{A}$ donné de l'équateur solaire : le fluide qu'il émet décrit, dans un système d'axes lié au Soleil, une courbe d'équations :

$\varphi=\varphi_{0}-\omega_{\odot}\left(1-R_{\odot} / r\right) t \approx \varphi_{0}-\omega_{\odot} t \quad r \cong R_{\odot}+V t$ soit :

$$
r=R_{\odot}+V \frac{\varphi_{0}-\varphi}{\omega_{\odot}}
$$

C'est l'équation d'une spirale d'angle $\psi$ est donné par :

$$
\operatorname{tg} \psi=\frac{r}{R_{\odot}} \frac{\omega_{\odot} R_{\odot}}{V} \sim \frac{1}{100}\left(\frac{r}{R_{\odot}}\right) .
$$

N.-B. - Dans (9), on a supposé $V(r) \approx$ Cte.

Raisonnant toujours dans ce référentiel solaire, le fluide totalement ionisé supposé infiniment conducteur doit obéir à l'équation (11) :

$$
\mathbf{E}+\mathbf{v} \times \mathbf{B}=\frac{1}{n e} \nabla p \approx 0 .
$$

Dans ce référentiel, $\mathbf{E}=0$ (pas de charge d'espace importante), d'où la condition :

$$
\mathbf{v} \times \mathbf{B}=0
$$

qui impose aux lignes de force d'être confondues avec la spirale (9).

On obtient alors aisément l'intensité du champ en tout point en écrivant la conservation du flux. Le résultat est :

$$
\begin{gathered}
B_{r}=B_{0}\left(\frac{R_{\odot}}{r}\right)^{2} \\
B_{\varphi}=B_{0} \frac{\omega_{\odot}}{V}\left(\frac{R_{\odot}}{r}\right)^{2}\left(r-R_{\odot}\right) .
\end{gathered}
$$

La figure 3 montre les variations de $B_{r}$ et $B_{\varphi}$.

(2) $R_{\odot}=7 \times 10^{5} \mathrm{KM}=1 / 214$ U.A.

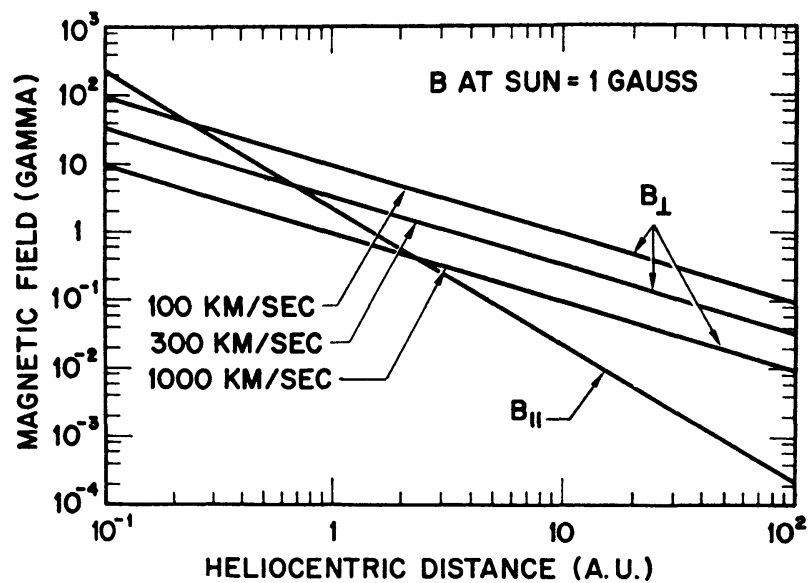

FIG. 3. - Valeur des composantes du champ magnétique parallèle au rayon vecteur et perpendiculaire au rayon vecteur, données par l'équation (11), [8]. Plusieurs vitesses d'expansion du vent ont été choisies.

Le changement référentiel solaire en référentiel absolu laisse le champ magnétique pratiquement inchangé, compte tenu de la faible vitesse relative, à cela près que le champ semble tourner uniformément avec le Soleil.

Nous sommes maintenant en mesure d'évaluer $\beta$ donné par (8).

$\mathrm{Au}$ voisinage de l'orbite terrestre, on voit, d'après les chiffres donnés en I.1.b, que :

$$
\begin{aligned}
& n k T \approx 30 \mathrm{eV} / \mathrm{cm}^{3} \\
& n m V^{2} \approx 5000 \mathrm{eV} / \mathrm{cm}^{3} \\
& B \approx 5 \text { gammas }\left({ }^{3}\right) \text { si } B_{0}=1 \text { gauss }
\end{aligned}
$$

donc :

$$
B^{2} / 2 \approx 60 \mathrm{eV} / \mathrm{cm}^{3}
$$

d'où :

$$
\beta=\frac{n m V^{2}}{\left(B^{2} / 2 \mu_{0}\right)} \gg 1 .
$$

Les formules (11) et (3) montrent que ce rapport se conserve au-delà de l'orbite terrestre. De la même manière, on peut calculer le rapport $\beta$ pour $r \simeq R_{\odot}$.

Il apparait alors que $\beta$ approche de 1 si $V \sim 100 \mathrm{~km} / \mathrm{s}$. On doit donc conclure que le vent solaire ne peut exister avec une vitesse d'expansion inférieure à $100 \mathrm{~km} / \mathrm{s}$ : le champ photosphérique agit en quelque sorte comme une « soupape de sûreté ».

Conclusion de cette section : il est prédit un champ magnétique qui, dans le plan de l'écliptique, est enroulé en spirale; son intensité est exprimée par (11), sa direction par (10). Au voisinage de l'orbite terrestre, $B \sim 5 \gamma$ et $\psi \sim 45^{\circ}$. Au voisinage de l'orbite de Jupiter $\left(r 4 / R_{\odot}=5,2\right), B \sim 0,7 \gamma$ et $\psi \sim 80^{\circ}$. En dehors de l'écliptique, les spirales sont enroulées sur des cônes ayant pour axe l'axe de rotation du Soleil. Dans tous les cas, il faut remarquer que chaque spirale est liée à un point donné de la photosphère, donc en un point donné de l'espace on observera des variations temporelles résultant de la rotation de la photosphère et de la non-homogénéité de celle-ci.

$\left.{ }^{3}\right) 1$ gamma $=10^{-5}$ gauss $=10^{-9}$ weber $_{/} \mathrm{m}^{2}$. 
b) Observations expérimentales du champ magnétique interplanétaire. - Faisons d'abord un bref rappel des principales mesures actuellement exploitées :

- Mariner II : Août 1962-novembre 1963, [12], Magnétomètre « Fluxgate » triaxial, $\pm 0,5 \gamma$;

- IMP 1 : Novembre 1963-mai 1964, [13], Magnétomètre «Fluxgate » triaxial, $\pm 0,25 \gamma$;

- Mariner IV : Novembre 1964-octobre 1965, [14], Magnétomètre « Fluxgate » triaxial, $\pm 0,5 \gamma$;

- Pioneer VI : Décembre 1965, [15], « Fluxgate » uniaxial $\left(54^{\circ} 45^{\prime}\right), 0,25 \gamma$, basculable en vol.

La figure 4 présente trois heures d'enregistrement fournies par Pioneer VI. On remarque que l'intensité du champ est à peu près constante, que son inclinaison

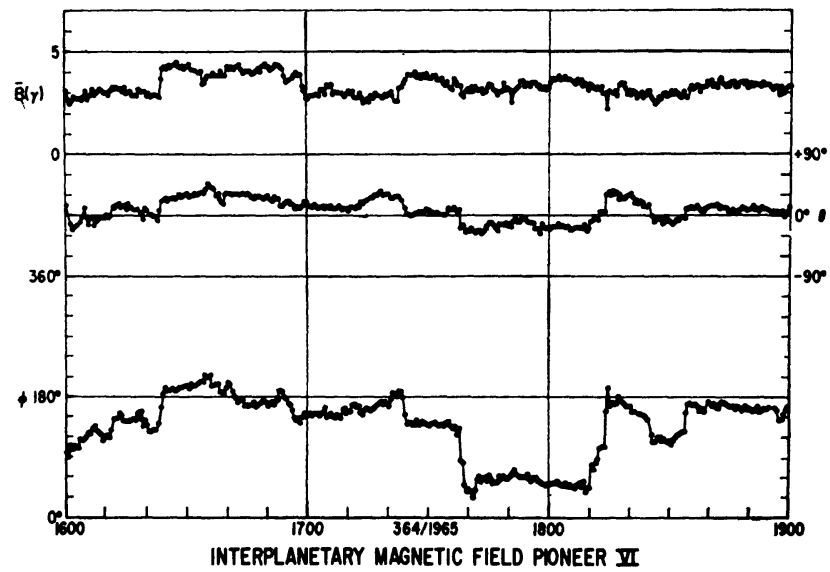

FIG. 4. - Mesure de l'intensité, de la direction du champ magnétique dans le vent solaire à bord de Pioneer VI, pendant 3 heures consécutives : $\varphi$ est l'angle entre $\mathbf{B}$ et la direction du Soleil ; $\theta$, l'angle entre $\mathbf{B}$ et l'écliptique [15].

par rapport à l'écliptique $\theta$ est presque nulle; l'angle $\varphi$ entre le champ et la direction du Soleil est voisin de $135^{\circ}$ ou $45^{\circ}$ (valeurs de la spirale décrite précédemment). Cependant on observe des discontinuités fréquentes, qui font penser à une structure «fibrée » du champ. Nous laisserons de côté ce problème de structure fine; une étape dans l'organisation des données a été franchie lorsque Ness et Wilcox [13] étudièrent systématiquement la polarité du champ; en découpant les mesures en intervalles de 3 heures, on peut attribuer à chaque intervalle un signe correspondant à la direction prédominante du champ pendant cet intervalle : + (direction opposée au Soleil), ou - (direction du Soleil). Il apparaît alors que l'on peut décomposer l'écoulement du temps en périodes successives de plusieurs jours pendant lesquelles le champ a une polarité presque constante. Finalement, la succession de ces « secteurs » se reproduit avec la périodicité de 27 jours correspondant à la rotation solaire.

Si l'on suppose que cet effet est dû à la corotation avec le Soleil de la structure spirale, on aboutit à une structure telle que celle de la figure 5 . La validité de cette hypothèse est confirmée :

1) Par une observation similaire pour les autres paramètres du vent solaire (densité, vitesse, etc.).

2) Par une excellente corrélation entre la succession

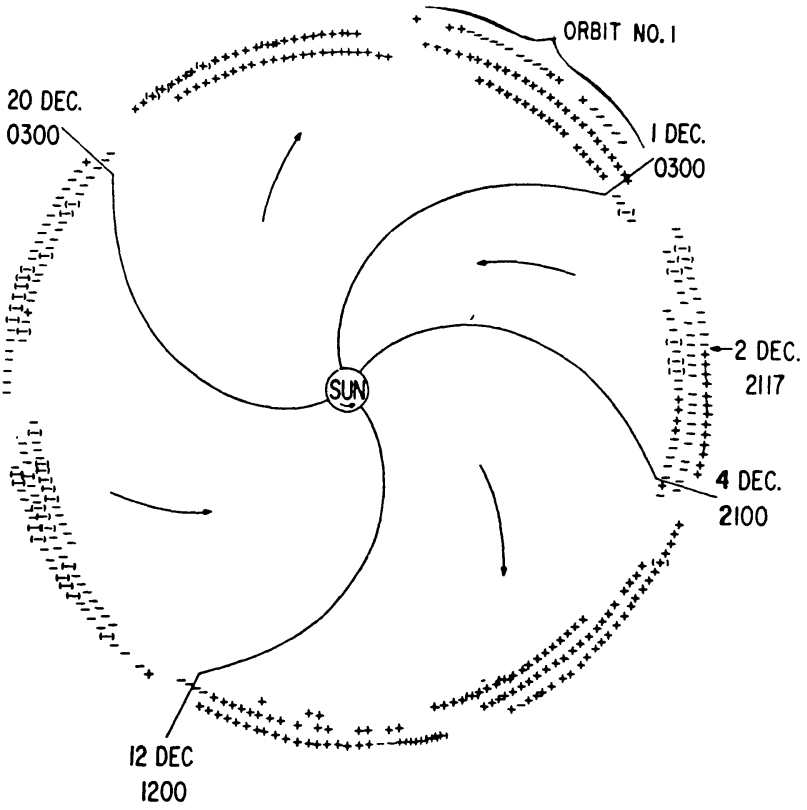

Fig. 5. - Polarité du champ magnétique dans le vent solaire, observée pendant trois rotations successives par IMP 1 [13].

des polarités mesurées par le satellite et les polarités moyennes du champ photosphérique (le décalage en temps résultant du temps de propagation est d'environ 5 jours) [13].

3) Par une comparaison directe entre les résultats simultanés de plusieurs satellites, ainsi Pioneer VI et IMP 3, séparés par 1,3 million de $\mathrm{km}$ (voir fig. 6 et réf. [16]).

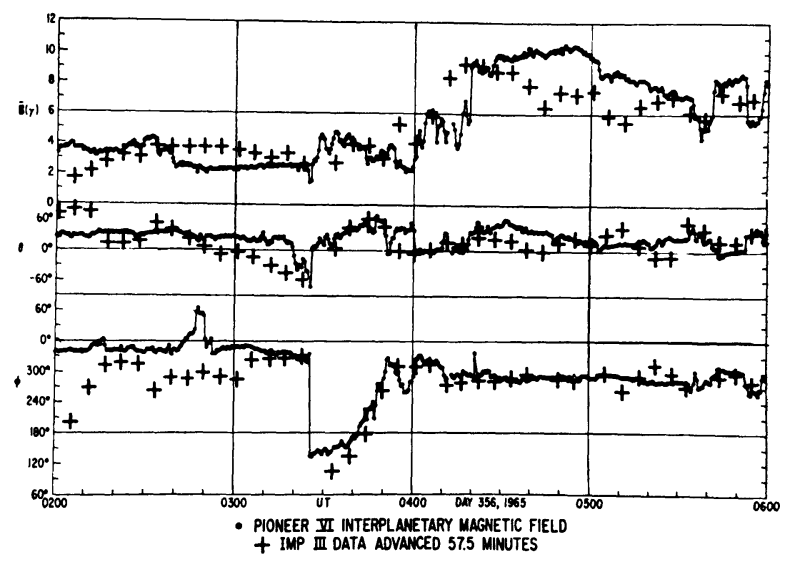

Fig. 6. - Mesures simultanées du champ magnétique interplanétaire par Pioneer VI et IMP 3. Le temps de rotation calculé du champ magnétique d'un satellite à l'autre est de $57,5 \mathrm{mn}$.

Pour conclure, les figures 7 et 8 donnent une information statistique relative à l'écart entre le champ réel et le champ théorique calculé en I.2.a). La première concerne la direction du champ, la seconde son intensité. Notons que l'existence d'une composante normale à l'écliptique statistiquement non nulle est actuellement controversée. On retiendra de ces figures que le modèle que nous avons décrit n'est qu'un modèle statistiquement satisfaisant. 


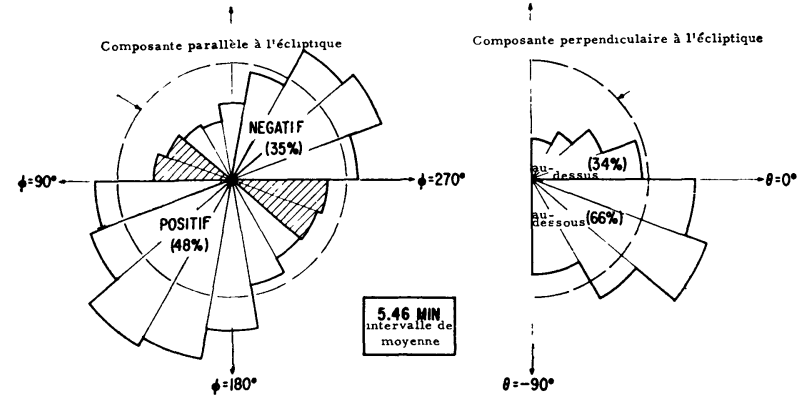

FIG. 7. - Statistique des orientations du vecteur champ magnétique dans le vent solaire, résultant des mesures de IMP 1. La figure indique le signe de la composante mesurée, $\varphi$ et $\theta$ sont la longitude et la latitude écliptiques. On remarque qu'en moyenne le champ est orienté suivant $\varphi=135^{\circ}$ ou $315^{\circ}$, direction de la spirale, et que ces mesures fournissent une composante normale prédominante vers le sud de l'écliptique.

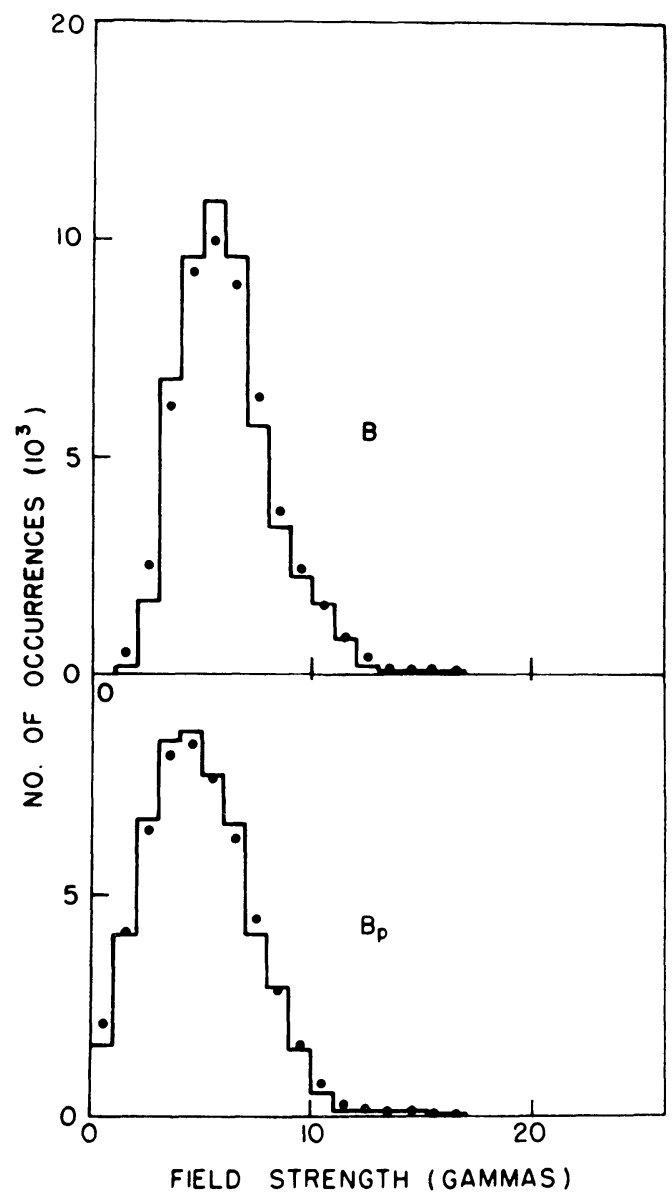

FIG. 8. - Distribution statistique du module du champ, au cours du vol de Mariner II : $B$ est le champ total ; $B_{P}$, la composante du champ suivant la direction de la spirale.

II. L'interaction vent solaire-magnétosphère du côté jour. Magnétopause et onde de choc stationnaire. - A l'heure actuelle, l'interaction du vent solaire avec deux « gros objets » est intensivement étudiée : la Terre d'une part, la Lune d'autre part. Dans un futur assez proche, on peut espérer que commencera l'étude de l'interaction avec Jupiter, laquelle, entre autres originalités, a l'intérêt de faire probablement intervenir le champ de gravitation de la planète. Enfin, l'interaction du vent avec Vénus fournirait un nouveau problème, car il est vraisemblable que le vent atteigne directement l'atmosphère supérieure de la planète (partiellement ionisée).

II.1. Physique de L'interagtion vent solaireCHAMP DIPOLAIRE TERRESTRE. - II.1.a) Le champ terrestre non perturbé. - En oubliant l'existence du vent solaire, le champ magnétique magnétosphérique provient de plusieurs sources :

1. Sources internes à la surface terrestre. - C'est la contribution prépondérante. Ce champ dépend d'un potentiel de $V_{I}^{*}$ développable en harmoniques sphériques (on prend habituellement pour origine le centre du géoïde terrestre) :

$$
\begin{aligned}
V_{I}^{*}= & R_{\oplus} \sum_{l=1}^{\infty}\left(\frac{R_{\oplus}}{r}\right)^{l+1} \\
& \sum_{m=0}^{l}\left(g_{l}^{m} \cos m \varphi+h_{l}^{m} \sin m \varphi\right) P_{l}^{m}(\cos \tilde{\theta})
\end{aligned}
$$

où $\varphi$ et $\tilde{\theta}$ sont respectivement la longitude et la colatitude géographiques et $R_{\oplus}$ le rayon terrestre moyen $(6371 \mathrm{~km})$. Les $P_{l}^{m}$ sont normalisés suivant la convention de Schmidt [17]. Les coefficients sont ajustés aux données expérimentales et publiés régulièrement dans la littérature (actuellement, développement limité à 120 coefficients).

Les termes $l=1$ donnent le moment dipolaire terrestre :

$M_{\oplus}=\frac{4 \pi}{\mu_{0}} R_{\oplus}^{3} \cdot \sqrt{g_{1}^{12}+h_{1}^{12}+g_{1}^{02}}=7,9 \times 10^{22} \mathrm{~A} \cdot \mathrm{m}^{2}$.

La dépendance en $r^{l+1}$ des potentiels dus aux harmoniques $l>1$ permet de restreindre à grande distance le potentiel à son seul terme dipolaire; il reste cependant à choisir une origine physiquement acceptable [18], on montre [19] que cette origine a actuellement les coordonnées suivantes :

$$
\begin{aligned}
& r_{0} \approx 350 \mathrm{~km} \\
& \tilde{\theta}_{0} \approx 6^{\circ} \\
& \varphi_{0} \approx 160^{\circ} \mathrm{E} .
\end{aligned}
$$

Enfin, la direction du vecteur $M_{0}$ est définie actuellement [19] par :

$$
\begin{aligned}
& \tilde{\theta}_{M}=11^{\circ} 27^{\prime} \\
& \varphi_{M}=69^{\circ} 30^{\prime} \mathrm{W} .
\end{aligned}
$$

2. Sources ionosphériques et magnétosphériques. - Ge sont les courants créés par les déplacements de matière ionisée dans la magnétosphère :

- Courants ionosphériques diurnes $S_{Q}$ et $S_{L}$ ou $S_{D}$;

- Courants résultant du mouvement du plasma magnétosphérique.

Les premiers circulent à très basse altitude, créant une perturbation au sol de l'ordre de quelques centaines de gammas, nous négligerons leurs effets à grande distance [20].

En ce qui concerne le plasma piégé, nous négligerons également son existence, il faut cependant se rendre compte que l'approximation ainsi faite est probablement non justifiée dans les régions externes. 
II.1.b) Méthodes de calcul de l'interaction gaz ionisé en mouvement-champ magnétique statique. - Ge sujet est relativement difficile : on consultera [19] qui contient une récapitulation d'ensemble.

On peut se représenter physiquement le phénomène de plusieurs manières :

1) Par référence à l'électromagnétisme classique : en approchant un plan infiniment conducteur d'un dipôle magnétique $M$, les courants induits dans le conducteur annulent complètement le champ dans le conducteur et au-delà; ils perturbent ipso facto le champ en avant du plan : on montre que tout se passe en avant du plan comme si on ajoutait au champ initial celui d'un dipôle fictif de même grandeur que $M$, et symétrique de celui-ci par rapport au plan mobile.

2) En négligeant le comportement collectif hydrodynamique du vent solaire, il est possible de déterminer le mouvement des particules individuelles, dans le champ magnétique qu'elles rencontrent. L'ensemble des trajectoires individuelles donne naissance à une distribution de courants équivalents qui sont responsables des perturbations du champ original. On peut alors procéder par une méthode d'itération pour trouver une solution self-consistante :

Champ d'ordre $n \longrightarrow$ Trajectoires d'ordre $n$

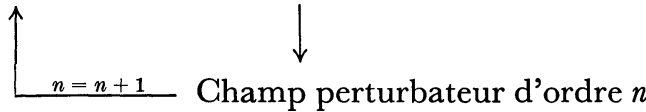

Cette méthode permet d'établir qu'il se forme une surface de discontinuité séparant le champ du milieu ionisé. Son épaisseur est :

$$
d \approx \frac{c}{\omega_{p}}=c \sqrt{\frac{m_{e} \varepsilon_{0}}{n e^{2}}}
$$

(ce qui donne pour le vent solaire : $d \sim 2 \mathrm{~km}$ !).

On montre que les particules sont réfléchies élastiquement sur cette surface, et que le champ magnétique subit une discontinuité donnée par :

$$
\frac{B_{F^{2}}}{2 \mu_{0}}=p_{\perp}
$$

où $p_{\perp}$ est la pression cinétique exercée par les particules chargées sur la surface.

3) En appliquant les équations d'équilibre macroscopique d'un gaz ionisé au voisinage d'une surface de discontinuité [11], [19], on peut alors écrire directement l'équation :

$$
\frac{B_{F^{2}}}{2 \mu_{0}}=p_{\perp}+\frac{b^{2}}{2 \mu_{0}}
$$

Il reste à évaluer $p_{\perp}$ le long de la surface de discontinuité.

Une évaluation inspirée des résultats de la méthode précédente conduit à faire pour la distribution des vitesses dans le vent solaire au voisinage de la surface de séparation l'hypothèse symbolisée par le graphique ci-dessous :

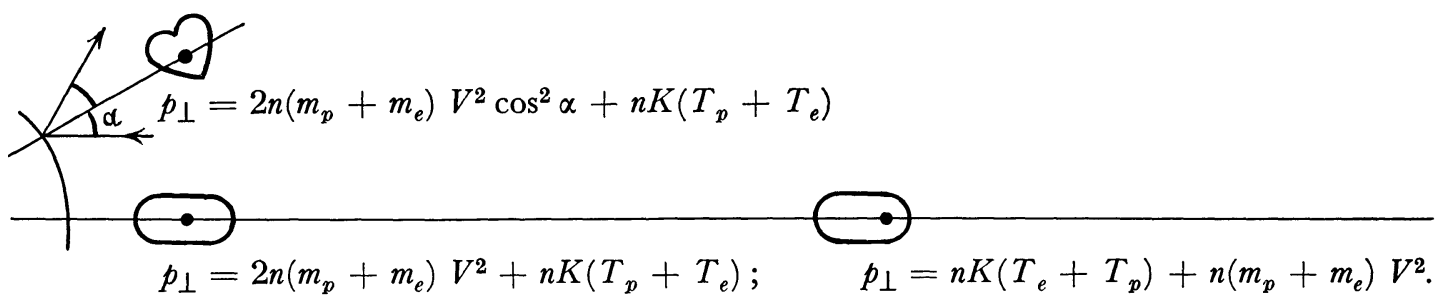

L'équilibre de la surface est alors décrit par l'équation semi-empirique :

$$
\begin{aligned}
\frac{B_{F^{2}}}{2 \mu_{0}}= & \frac{b^{2}}{2 \mu_{0}}+n_{e} K T_{e}+n_{p} K T_{p}+ \\
& 2 n\left(m_{e}+m_{p}\right) V^{2} \cos ^{2} \alpha \simeq 2 n m_{p} V^{2} \cos ^{2} \alpha .
\end{aligned}
$$

L'équation (15) peut être considérée comme une équation différentielle de la surface de séparation ou « magnétopause », si toutefois $B_{F}$ peut être relié au champ non perturbé (dipolaire) $B_{D}$.

Si la surface de séparation est plane, on a simplement :

$$
\left[B_{F}\right]=2 B_{D} .
$$

Si la surface n'est pas plane, la relation dépend de la forme de la surface, et n'est pas connue a priori. Diverses méthodes ont été employées pour lever cette difficulté. Nous renvoyons le lecteur aux références [19], [21], [22].

4) Enfin la méthode qui actuellement fournit le plus d'information sur l'interaction est l'application des équations de la dynamique des gaz ionisés. Nous y reviendrons au $\S$ II.1.d). Gette méthode ne remet pas en cause la forme de la magnétopause que l'on déduit de l'équation (15); c'est pourquoi nous allons tout de suite exposer les résultats de la méthode 3 , et les comparer aux données expérimentales [19], [21], [22].

II .1.c) Forme de la magnétopause côté jour. — La figure 9 présente, calculées par plusieurs méthodes indépendantes, les intersections de la magnétopause par le méridien magnétique. (N.B. - En supposant que le vent solaire « souffle » perpendiculairement à l'axe du dipôle terrestre.)

L'unité de longueur est :

$$
L_{f}=\left(\frac{\mu_{0} M_{\oplus}^{2}}{16 \pi^{2} n m_{p} V^{2}}\right)^{1 / 6} \approx 8 \text { à } 12 R_{\oplus} .
$$

Si dans la formule (15) on fait $B_{F} \equiv 2 B_{D}$, c'est-àdire si on néglige les perturbations du champ dipolaire à l'intérieur de la magnétopause, $L_{f}$ est la distance entre le point subsolaire de la magnétopause et l'origine du dipôle.

La figure 10 présente les résultats transmis par la sonde Pioneer VI qui s'éloigne de la Terre dans une direction à peu près perpendiculaire à celle du Soleil. On remarque que, à l'intérieur de la magnétopause, le champ est nettement supérieur à la valeur dipolaire, puis apparaît $(13 \mathrm{~h})$ une première discontinuité, la magnétopause, ensuite, et jusqu'à $17 \mathrm{~h}$, Pioneer VI traverse une zone extrêmement turbulente; à $17 \mathrm{~h} 10$, il franchit une seconde discontinuité et pénètre dans le vent solaire. 


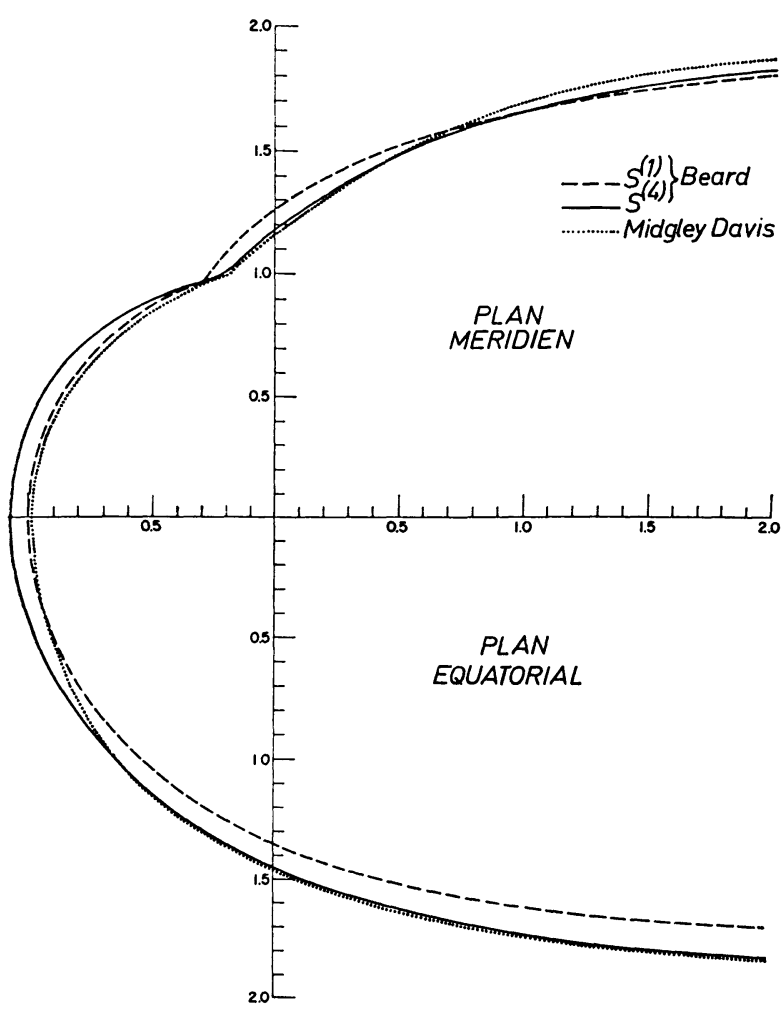

Fig. 9. - Forme de la cavité magnétosphérique dans un demi-méridien magnétique et dans un demi-plan équatorial magnétique. Les différentes courbes correspondent à différentes méthodes de calcul ; l'unité de longueur est $L_{f}$ (formule (16)).

Une étude systématique des deux discontinuités a été faite, par exemple, par IMP 1. La figure 11 compare les résultats obtenus avec les formes théoriques calculées pour des paramètres moyens du vent solaire. On voit que l'accord est très satisfaisant. Nous allons maintenant dire quelques mots de la seconde discontinuité.

II.1.d) La structure de choc stationnaire en amont de la magnétosphère. - La théorie de l'interaction exposée en II.1.b) est extrêmement primitive, car elle néglige tout comportement collectif du vent solaire autre que celui donné par les équations de Maxwell macroscopiques; l'évaluation de la pression cinétique exercée sur la magnétopause résulte alors de la réflexion spéculaire calculée pour chaque particule individuelle. Dans ces conditions, $n$ et $T$ sont, au voisinage de la magnétopause, celles du vent solaire non perturbé.

Si l'on fait l'hypothèse, au contraire, que le vent solaire continue, à grande distance du Soleil, à posséder un comportement fluide, le problème est celui de l'interaction d'un gaz ionisé avec un objet - la magnétosphère - en mouvement rapide par rapport à lui. On montre [23], [24] que :

1) Le problème se sépare en deux sous-problèmes : d'une part, déterminer la magnétopause; d'autre part, connaissant la magnétopause, calculer la structure du gaz ionisé en amont. On peut donc procéder par approximations successives à partir de la magnétopause « d'ordre zéro » calculée en II.1.c).

2) La pression cinétique choisie en (15) est une

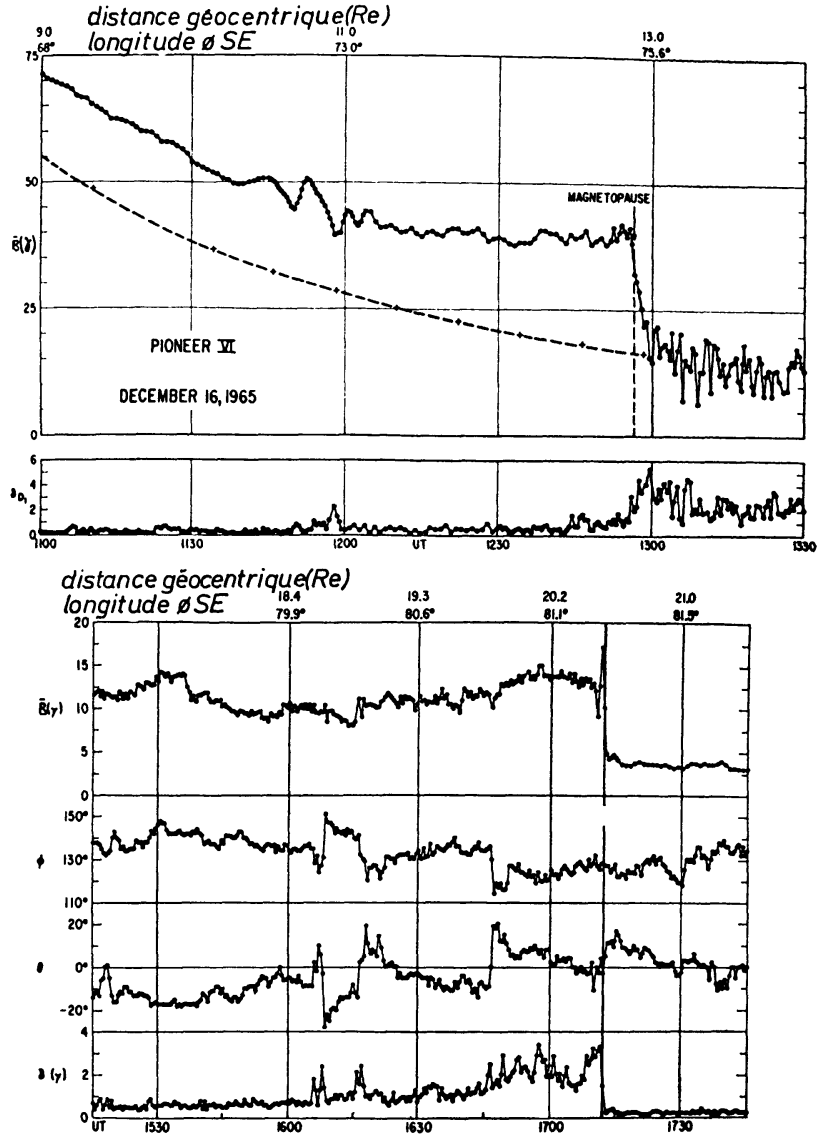

Fig. 10. - Amplitude et turbulence du champ magnétique mesuré lors de l'éloignement de la sonde héliocentrique Pioneer VI. La grandeur $\delta$ donne une idée de la turbulence en amplitude :

$$
\delta B=\sqrt{(B-\bar{B})^{2}} .
$$

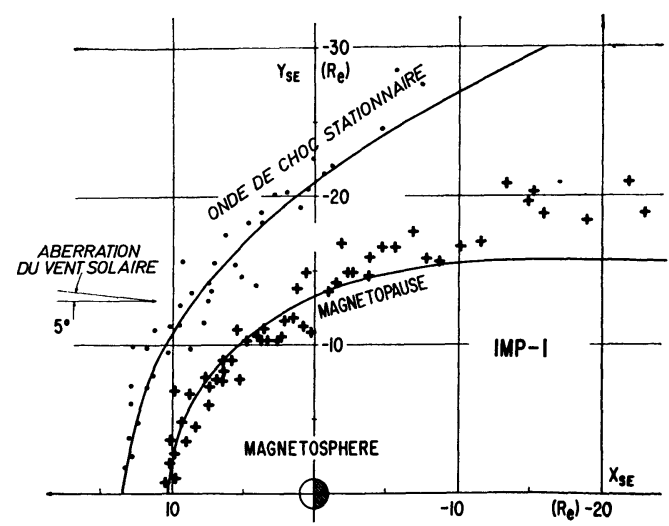

Fig. 11. - Positions, identifiées par IMP 1, de l'onde de choc stationnaire et de la magnétopause, comparées aux formes calculées théoriquement. On se reportera à $[24]$, p. 420-421, pour des résultats plus exhaustifs (Explorer XXXIII).

excellente approximation, bien que la température, la pression et la direction de mouvement du vent solaire soient très différentes des valeurs non perturbées.

3) Il se forme une onde de choc stationnaire en amont de la magnétopause, ceci étant dû au fait que la vitesse relative est grande aussi bien devant la vitesse 
du son $V_{s}$ que celle d'Alfven $V_{\mathrm{A}}$ : les nombres de Mach sont :

$M_{s}=V / V_{s} \cong 2-3 \quad$ et $\quad M_{\mathrm{A}}=V / V_{\mathrm{A}} \cong 3-10$.

4) La structure du fluide entre l'onde de choc et la magnétopause peut se calculer en utilisant les équations des gaz neutres; on en déduit alors la structure du champ magnétique.

Les figures 12 et 13 fournissent les résultats du calcul de la densité, de la température et de la direction

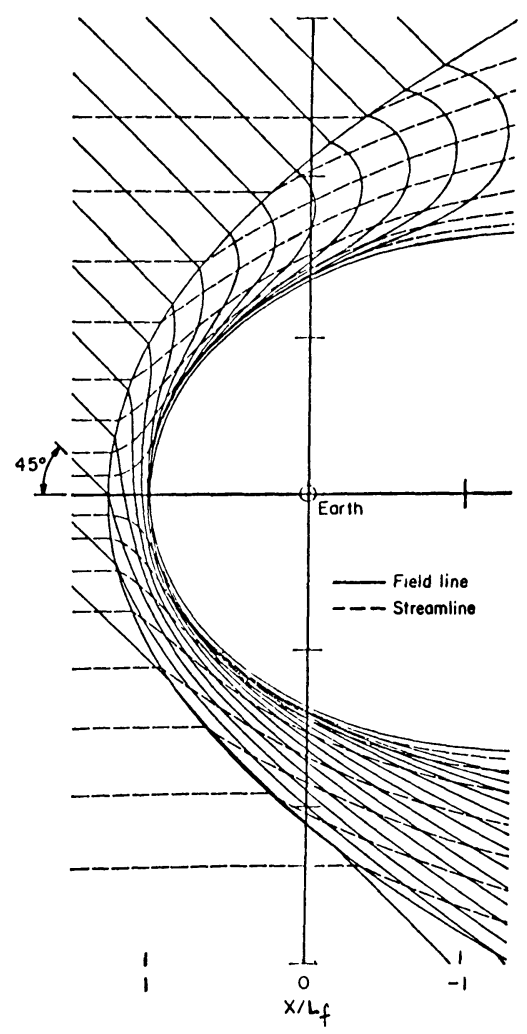

FIG. 12. - Écoulement du vent solaire le long de la magnétopause et direction du champ magnétique interplanétaire. Le plan de figure est le plan qui contient la direction d'écoulement du vent solaire, la direction du champ magnétique dans le vent solaire, et le centre de la Terre [23]. En dehors de ce plan, les lignes de force du champ ne sont pas planes. L'approximation de la forme de la magnétopause est expliquée à l'occasion de la figure 13.

moyenne du champ magnétique, tandis que la figure 14 rassemble des déterminations expérimentales de la direction moyenne du champ. Ici encore, on constate un accord satisfaisant. Pour le calcul théorique ayant conduit aux résultats des figures 12 et 13, la magnétopause est remplacée par une surface de révolution ayant pour méridienne l'intersection équatoriale de la magnétopause. De sorte que les résultats obtenus ne sont quantitatifs qu'au voisinage du plan de l'écliptique.

II .1.e) Conclusions. - Les équations de la dynamique des fluides neutres en écoulement supersonique appliquées au vent solaire expliquent de façon satisfaisante les principaux aspects observés de l'interaction ventchamp magnétique terrestre, du côté « jour » de la magnétosphère.

Cette théorie ne fournit cependant pas de résultats utilisables quant à la direction antisolaire : elle prédit
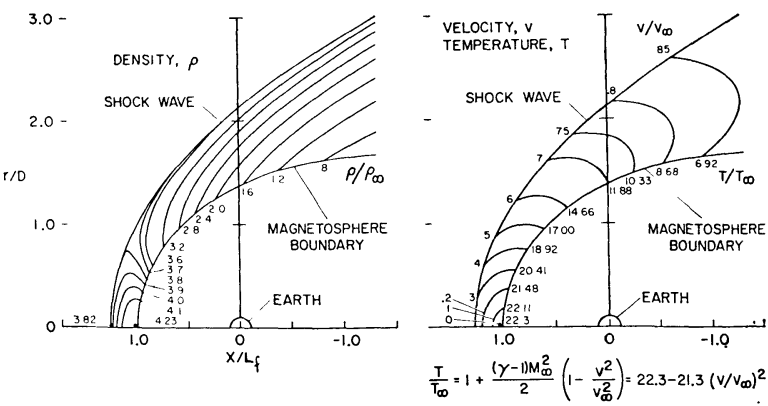

FIG. 13. - Lignes d'équidensité, et lignes $V / V_{\infty}=$ Cte entre la magnétopause et l'onde de choc stationnaire [23]. Les quantités $V_{\infty}, p_{\infty}, M_{\infty}, T_{\infty}$ sont relatives au vent solaire non perturbé. La forme de la magnétopause réelle est remplacée par une surface de révolution ayant pour méridienne l'intersection équatoriale de la magnétopause réelle.

On voit que les paramètres du plasma le long de la magnétopause diffèrent considérablement de leur valeur dans le vent solaire. Néanmoins, la pression cinétique est à peu près celle du vent non perturbé.
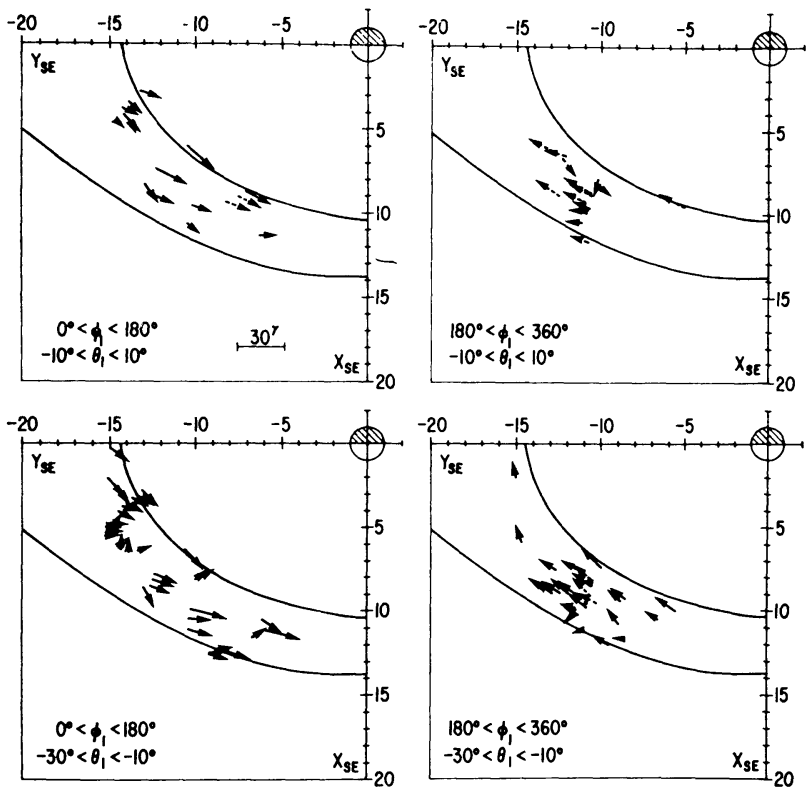

FIG. 14. - Projections du vecteur champ magnétique entre l'onde de choc stationnaire et la magnétopause, mesurées à bord de IMP 1 et IMP 2 [25]. La flèche a une longueur proportionnelle à l'amplitude du vecteur. L'axe $X_{\mathrm{SE}}$ est l'axe Terre-Soleil, l'axe $Y_{\mathrm{SE}}$ lui est perpendiculaire et contenu dans le plan de l'écliptique. Les distances sont comptées en $R_{\oplus}$.

une magnétopause en forme de cylindre infini, ainsi qu'une onde de choc asymptotiquement conique. Ceci est dû en fait à l'application des équations du gaz neutre mentionnée en II.1 d). Mais dans la direction antisolaire, le vent solaire s'écoule parallèlement à la magnétopause, de sorte que celle-ci est déterminée par les pressions $n K T$ et $B^{2} / 2 \mu_{0}$ du fluide, ce qui requiert les équations magnétodynamiques.

Nous avons donc actuellement à recourir aux seules observations expérimentales pour la région antisolaire. G'est pourquoi nous examinerons cette région simultanément avec l'étude expérimentale du champ magnétosphérique. 
III. Structure du champ magnétosphérique et forme de la magnétosphère antisolaire. - III .1 a) STRUCTURE THÉORIQUE DU GHAMP. - Pour simplifier, nous écarterons une difficulté : l'orientation relative du moment dipolaire terrestre et de la direction d'incidence du vent solaire change constamment, soit par la rotation diurne (et le mouvement orbital) de la Terre, soit par suite des variations du vent solaire lui-même. L'amplitude maximale de cette variation est au cours de l'année $\pm 30^{\circ}$ environ. On consultera [19] pour une discussion de cet effet. Dans la suite, nous supposerons que les deux directions sont perpendiculaires.

Une fois déterminées la forme de la magnétopause et la distribution des courants, il est simple de calculèr le champ perturbateur qu'ils créent à l'intérieur de la magnétopause. Usuellement, on représente ce champ par son développement multipolaire en coordonnées géomagnétiques :

$$
\begin{aligned}
& V_{\mathrm{ext}}^{*}\left(r, \tilde{\theta}_{m}, \varphi_{m}\right)=R_{\oplus} \sum_{l=1}^{\infty}\left(\frac{r}{R_{\oplus}}\right)^{l} \\
& \quad \sum_{m=0}^{l}\left(\tilde{g}_{l}^{m} \cos m \varphi_{m}+\tilde{h}_{l}^{m} \sin m \varphi_{m}\right) P_{l}^{m}\left(\sin \tilde{\theta}_{m}\right) .
\end{aligned}
$$

Les coefficients ont été calculés de façon indépendante par Mead [26] et Midgley [27]. Les figures 15 a, b présentent quelques aspects du champ total des potentiels (12) et (16).

On remarquera que les lignes de force ne sont pas planes; par ailleurs, le calcul de $B$ montre que certaines lignes admettent deux valeurs minima de $B$ séparées par un maximum secondaire. Enfin, les lignes issues des régions polaires $\left(\theta_{m} \geqslant 82^{\circ}\right.$ pour $\left.L_{f}=10 R_{\oplus}\right)$ traversent le plan équatorial côté nuit, même celles qui naissent dans le méridien de midi.

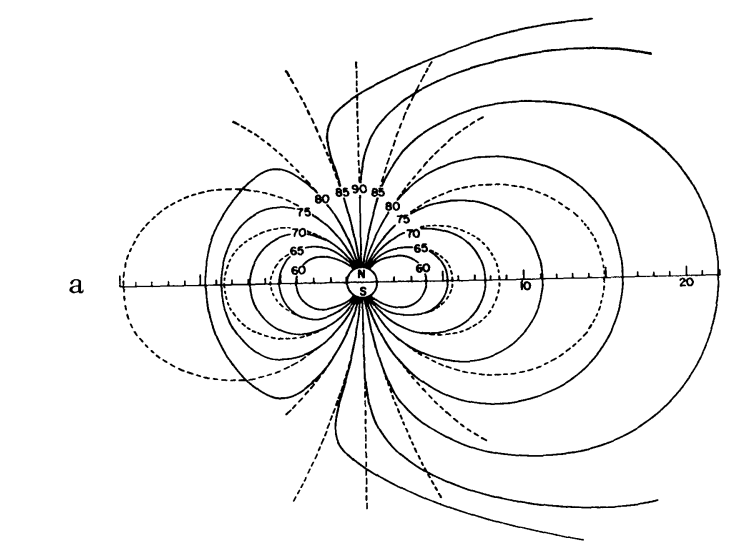

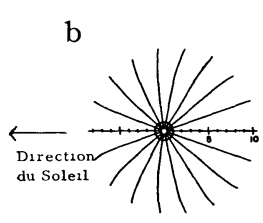

$\lambda_{\text {mII }}=75^{\circ}$

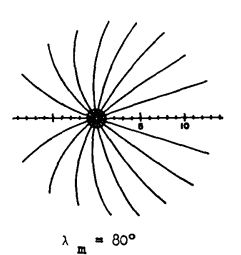

FIG. 15.

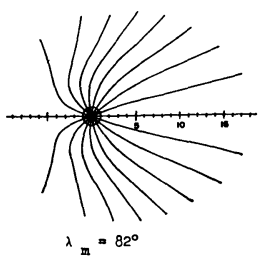

$x_{m}=82^{\circ}$

a) Structure des lignes de force calculées dans le méridien magnétique midi-minuit pour une incidence normale du vent et $L_{f}=10 R_{\oplus}$. Les pointillés représentent le champ dipolaire terrestre non perturbé. Les chiffres indiquent la latitude magnétique du pied de la ligne de force.

b) Projection sur le plan équatorial magnétique des lignes de force issues des latitudes magnétiques indiquées et de longitudes équiespacées de $20^{\circ}$ (réf. J. Geophys-res., 1964, 69, 1181-1195).
III.1 b) DÉtermination EXPÉRIMENTALE DE LA STRUCTURE DU GHAMP GOTÉ JOUR. - La figure 16 montre une mesure du champ magnétique faite à bord d'Explorer XII, à proximité de l'équateur géomagnétique et du méridien de midi géographique. On constate que l'addition des harmoniques (16) améliore la représentation du champ dans les régions externes; remarquer aussi vers $R=3,5 R_{\oplus}$ une forte dépression du champ, qui est due vraisemblablement au gaz ionisé confiné dans cette région de la magnétosphère. Nous ne parlerons pas ici de ce problème qui est cependant actuellement en plein développement.

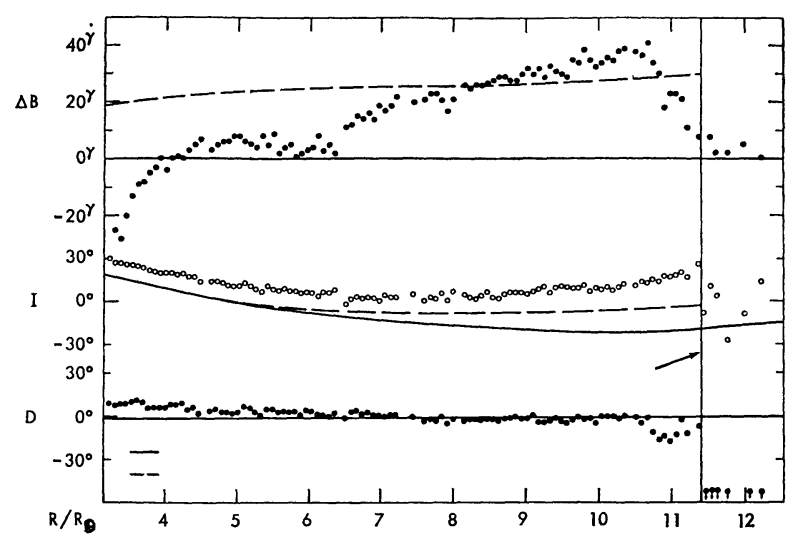

FIG. 16. - Écart $\Delta B$ entre le champ d'origine interne calculé par le développement multipolaire " Jensen Cain 1962 " et le champ mesuré au cours d'une orbite du satellite Explorer XII. La courbe en traits interrompus est calculée par le développement multipolaire de Mead du champ d'origine externe. $I$ est l'inclinaison et $D$ la déclinaison. La figure indique également la distance géocentrique, mesurée en rayons terrestres.

Une détermination empirique des lignes de force « moyennes » a été faite à partir de la compilation des résultats de IMP 1, 2, 3 par Fairfield [28]. La figure 17 donne un exemple des résultats. Qualitativement, l'accord avec le modèle de Mead est satisfaisant. On note pourtant un sévère désaccord dans la direction antisolaire, sur lequel nous allons nous étendre maintenant.

III.1 c) STRUGTURE DE LA MAGNÉTOSPHÈre ANTISOLAIRE. - Nous avons reproduit figure 18 les résultats obtenus par le satellite IMP 1, au moment où son apogée était voisin de la direction antisolaire. Ces résultats font apparaître :

1) Que le champ magnétique est aligné suivant la direction antisolaire.

2) Qu'il possède deux orientations (solaire et antisolaire) séparées par une région où le champ est pratiquement nul. Cette structure s'étend jusqu'à des distances de plusieurs centaines de $R_{\oplus}$ [30], constituant la « queue » de la magnétosphère. Cette région est une « cible » difficile à atteindre pour un satellite excentrique de sorte que la détermination de sa structure, et a fortiori celle de l'origine de cette structure, progressent très lentement. Les lignes de force présentes dans la queue se referment sur les calottes polaires. Ce qui permet, connaissant le diamètre de la queue, de calculer l'ordre de grandeur du champ magnétique en fonction de la distance (conservation du flux). La 


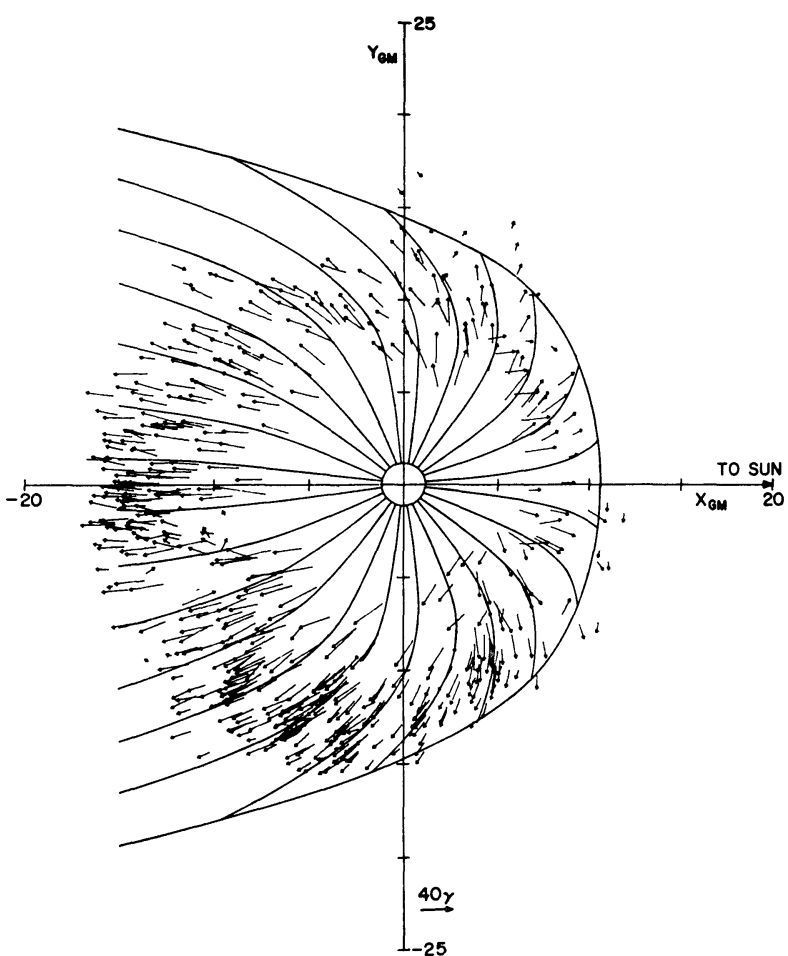

FiG. 17. - Projection sur l'équateur magnétique des vecteurs B moyennés sur une heure : Les lignes continues représentent la magnétopause et les projections de 24 lignes de force assujetties aux données par calcul numérique [28]. Les mesures compilées sont relatives à l'hémisphère sud. Les distances sont exprimées en $R_{\oplus}$.

figure 19 résume plusieurs séries de mesures relatives à cette intensité.

Pour terminer, remarquons que l'équilibre d'une telle structure ne peut être assuré que par une pression de plasma existant au niveau de la couche neutre, ce que confirment les observations expérimentales [31]. L'origine de ce plasma et en général le mécanisme assurant la formation de la queue ne sont pas connus.

IV. Le champ magnétique à proximité de la Lune. - Les mesures actuellement publiées sur l'environnement magnétique de la Lune ont été obtenues sur Explorer XXXIII (orbite très excentrique terrestre) et Explorer XXXV (orbite sélénocentrique). Il est maintenant établi que le champ magnétique est celui du vent solaire ou, lorsque la Lune pénètre dans la queue de la magnétosphère, celui de la queue. La présence de la Lune apporte seulement des perturbations assez faibles dans la direction opposée à l'écoulement du vent solaire. Le calcul théorique de ces perturbations n'est pas actuellement achevé, par suite d'incertitudes sur le mécanisme de l'interaction Lunevent solaire. Inversement, les observations magnétiques dans l'environnement lunaire donnent accès à quelques propriétés du corps lunaire.

IV.1. PropriÉtÉs INTRINsèQUes DE LA LUNE. IV.1 a) Limite supérieure du moment magnétique propre. Les meilleures conditions d'observation sont fournies lorsque la Lune traverse la queue de la magnétosphère, dont la structure est relativement stationnaire, et où on attend une interaction plasma-Lune négligeable. En effet, la vitesse relative est de $450 \mathrm{~km} / \mathrm{s}$ dans le vent solaire, et de $1 \mathrm{~km} / \mathrm{s}$ dans la queue. Une telle traversée
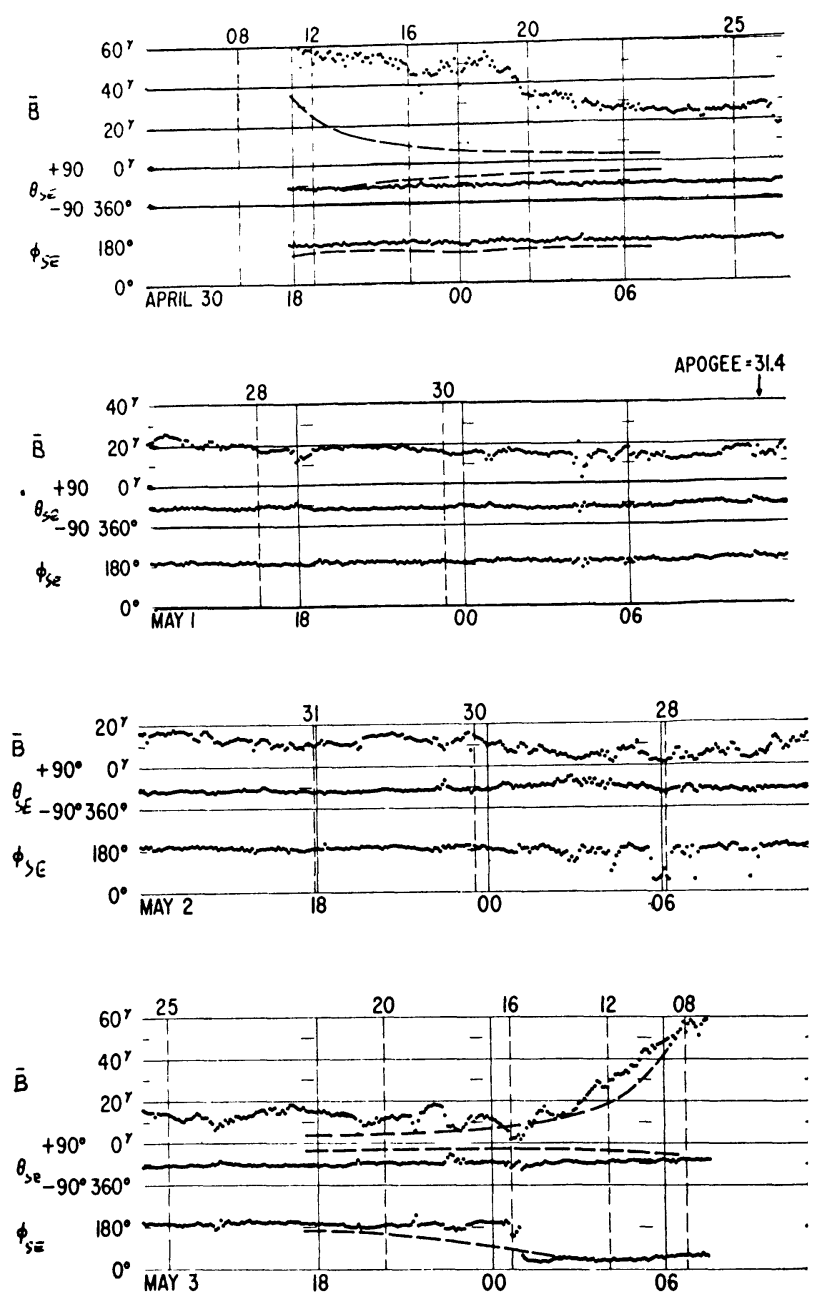

FIG. 18. - Identification de la queue de la magnétosphère par IMP 1 en mai 1964. Le satellite est au voisinage du méridien de minuit. La figure donne le module et les cosinus directeurs $\theta$ et $\varphi$ du vecteur $B$ dans le référentiel écliptique. La distance géocentrique du satellite est indiquée (en $R_{\oplus}$ ). On remarquera que le champ est à peu près parallèle à la direction antisolaire, qui est la direction $\theta=0, \varphi=0$. Également, on notera l'annulation brusque suivie de renversement du sens du champ le 3 mai à $16 R_{\oplus}$ : IMP 1 traverse la couche neutre.

a été observée, par exemple en octobre 1967 par Explorer XXXV. La Lune a même directement traversé la couche neutre [33].

Le champ mesuré est :

$$
\mathbf{B}=\mathbf{B}_{\oplus}+\mathbf{B}_{\Im}+\alpha \mathbf{B}_{\oplus}
$$

où $\mathbf{B}_{\oplus}$ désigne le champ magnétique dans la queue, $\mathbf{B}_{\S}$ un éventuel champ magnétique lunaire, et $\alpha \mathbf{B}_{\oplus}$ un éventuel champ de polarisation qui existe si la perméabilité magnétique de la Lune n'est pas égale à un (la constante $\alpha$ dépend de la répartition des masses magnétiques éventuelles à l'intérieur de la Lune). En désignant par $\mathbf{B}^{+}$et $\mathbf{B}^{-}$les champs mesurés au-dessus et au-dessous de la couche neutre, on a :

$$
\begin{aligned}
\mathbf{B}_{3} & =\frac{1}{2}\left(\mathbf{B}^{+}+\mathbf{B}^{-}\right) \\
\alpha \mathbf{B}_{\oplus} & =\frac{1}{2}\left(\mathbf{B}^{+}+\mathbf{B}^{-}\right)-\mathbf{B}_{\oplus}
\end{aligned}
$$

$\mathbf{B}_{\oplus}$ est donné par exemple par la figure 19 . 


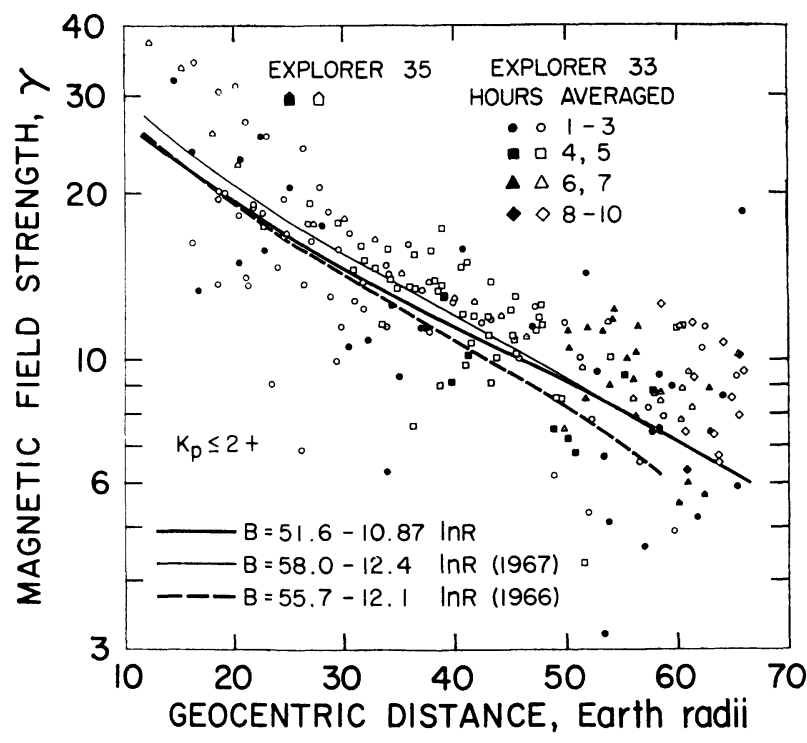

FIG. 19. - L'intensité du champ magnétique dans la queue de la magnétosphère, d'après les résultats d'Explorer XXXIII et Explorer XXXV. Les chiffres qui figurent à droite des symboles sont le nombre d'heures pendant lequel les données sont moyennées pour fournir le point expérimental correspondant [29].

Explorer XXXV trouve $\left|\mathbf{B}_{3}\right|<0,5$ gamma à une altitude sélénocentrique de $2400 \mathrm{~km}\left(\sim 1,4 R_{\Im}\right)$ quelle que soit la longitude lunaire (l'inclinaison de l'orbite sur l'écliptique lunaire est d'environ $14^{\circ}$ ). De même $\left|\alpha \mathbf{B}_{\oplus}\right| \leqslant 0,5$ gamma.

Ceci permet de placer une limite supérieure au moment dipolaire lunaire :

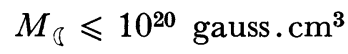

ou

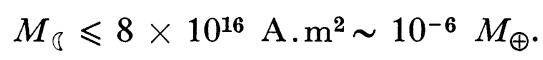

IV.1 b) Perméabilité magnétique de la Lune. - Si la Lune est une sphère homogène, placée dans un champ uniforme $B_{\oplus}$, elle prend un moment dipolaire induit $\mathbf{M}_{\mathbb{Q}}^{*}$ :

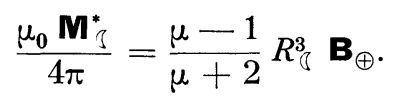

On obtient immédiatement une limite supérieure pour la perméabilité relative $\mu$ :

$$
\mu \leqslant 1,8 \text {. }
$$

Cependant, si la Lune est inhomogène, par exemple si pour $r \leqslant R_{c}$ la température est supérieure au point de Curie, la relation entre $\mathbf{M}_{\mathbb{B}}^{*}$ et $\mathbf{B}_{\oplus}$ fait intervenir la perméabilité $\mu$ de la région externe et $R_{c}$, de sorte qu'aucune limite supérieure ne peut être dans ce cas fixée pour $\mu$.

IV.1 c) Conductivité électrique de la Lune. - Des indications sur la conductivité de la Lune peuvent être déduites de l'observation de la vitesse de diffusion à travers la Lune des irrégularités du champ externe. En effet, une perturbation magnétique ne peut s'établir dans un milieu de perméabilité $\mu \mu_{0}$, conductivité $\sigma$ que suivant l'équation :

$$
\frac{\partial B}{\partial t}=\frac{1}{\mu \mu_{0} \sigma} \Delta B
$$

Pour s'établir sur une distance de l'ordre de $D$, le temps est de l'ordre de :

$$
\tau=\mu \mu_{0} \sigma D^{2} .
$$

La figure 20 présente deux mesures du champ magnétique, l'une est due à Explorer XXXV en orbite circumlunaire, l'autre à Explorer XXXIII qui, à cette

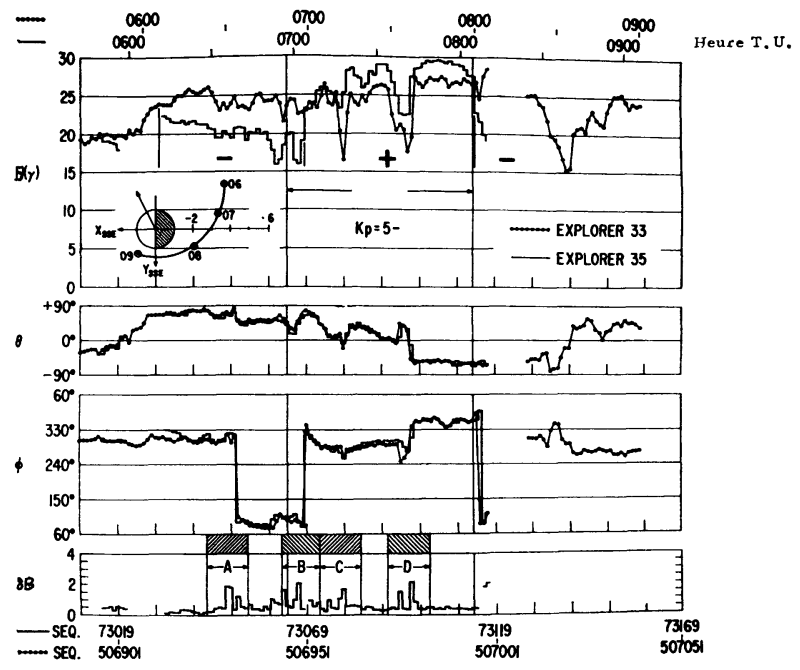

FIG. 20. - Mesures simultanées par Explorer XXXIII et Explorer XXXV du champ magnétique. Les coordonnées sont les coordonnées écliptiques solaires. $\delta B$ est la dispersion des mesures de $B$, soit $\sqrt{(B-\bar{B})^{2}}$ (chaque point de $B$ est la moyenne de 16 mesures consécutives). La position d'Explorer XXXV par rapport à la I une est représentée en projection sur l'écliptique. En haut à droite, figure la projection de l'orbite sur un plan perpendiculaire à la direction Soleil-Lune. La figure donne des résultats acquis le 13 septembre 1967, alors qu'Explorer XXXIII se trouve très proche de la Lune, une distance d'environ $60 R_{3}$. On voit que les discontinuités du champ magnétique dans le vent solaire (Explorer XXXIII) diffusent librement à travers la Lune (Explorer XXXV) [35].

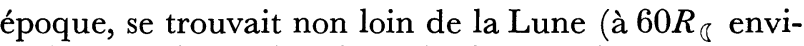
ron). On voit que les discontinuités du champ magnétique ont pu diffuser librement à travers la Lune. Ce qui signifie que l'on doit avoir, $V$ désignant la vitesse du vent solaire :

$$
\tau \leqslant 2 R_{\triangleleft} / V \sim 10 \mathrm{~s} .
$$

Si l'on fait l'hypothèse que la Lune est homogène, on déduit, en utilisant (19), une limite supérieure pour la conductivité de la Lune :

$$
\sigma \lesssim .10^{-5} \mathrm{mho} / \mathrm{m} \text {. }
$$

La Lune est donc un mauvais conducteur, ce qui signifie que son intérieur est probablement assez froid [32].

IV .2. INDIGATIONS SUR L'INTERAGTION DU VENT SOlaire aveg la Lune. - IV.2 a) Données expérimentales. - Les mesures magnétiques obtenues à bord des satellites cités précédemment ont montré clairement qu'il n'existe aucune perturbation magnétique en amont de la Lune dans le vent solaire [32], [34].

Par contre, en aval, un satellite circumlunaire observe une perturbation magnétique qui a la signature suivante $:+-(+)-+$. Le signe + signifie une 
augmentation du champ, le signe - une diminution. Le signe $(+)$ indique la région d'ombre postlunaire, elle est donc bornée par des perturbations « de pénombre ». Ce phénomène se voit très nettement sur la figure 21. On ne relève aucun indice d'une onde de
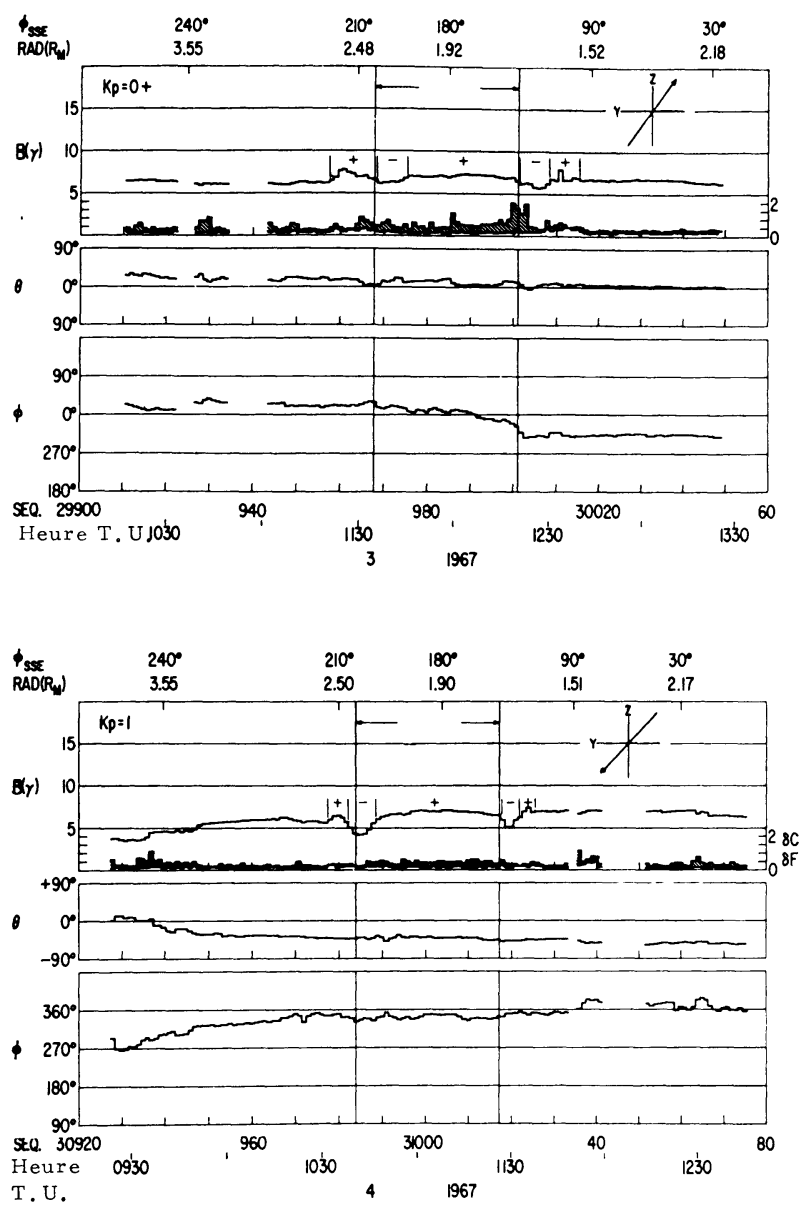

FIG. 21. - Mesures magnétiques recueillies par Explorer XXXV en août 1967. Le référentiel dans lequel est donnée la direction du champ est le référentiel solaire écliptique sélénocentrique. L'angle $\varphi_{S S E}$ est l'angle entre la direction Soleil-Lune et la direction de l'aposélène. RAD est la distance sélénocentrique, exprimée en multiples du rayon lunaire : $R_{\mathbb{Q}}=1738 \mathrm{~km}$ [34].

choc comparable à ce qui se produit en avant de la magnétopause. Par ailleurs, le dépouillement préliminaire des résultats obtenus par des sondes à plasma indique également l'absence de perturbation en amont, tandis qu'en aval existe une cavité dépourvue de plasma bordée par des zones de densité décroissante (pénombre).

IV .2 b) Interprétation théorique. - Un modèle définitif de l'interaction Lune-vent solaire n'a pas encore été établi actuellement.

On doit tout d'abord remarquer que la Lune est un objet conducteur en mouvement à la vitesse $V$ par rapport au champ magnétique qui imprègne le vent solaire. Il s'ensuit qu'en régime stationnaire des courants circulent à l'intérieur de la Lune sous l'effet du champ d'induction $\mathbf{E}=-\mathbf{V} \times \mathbf{B}$; ces courants à leur tour modifient la structure du champ magnétique excitateur. En particulier, si la conductivité est grande, le champ en avant de la Lune peut être assez intense pour qu'il se crée une magnétopause, donc une onde de choc. En fait, l'expérience montre que ceci n'a pas lieu, ce qui confirme la très faible conductivité de la Lune. Pour une discussion approfondie, le lecteur pourra se reporter à [36]. Les perturbations magnétiques résultent donc essentiellement des perturbations apportées à l'écoulement du vent solaire.

Tout objet réfléchissant en mouvement supersonique dans un fluide est précédé d'une structure de choc; on est donc conduit à supposer que la Lune, qui reçoit directement le vent solaire, est totalement absorbante. Il reste à calculer l'écoulement du vent en arrière de cet obstacle. Whang [34] et Ness ont calculé un sillage en traitant le vent solaire comme un gaz sans collision, dont les mouvements individuels sont déterminés par le champ magnétique dans le sillage; réciproquement, le champ dépend de ces mouvements. Cette méthode est analogue à celle exposée en II .1 b), 2). Les résultats reproduisent qualitativement la « signature » + $(+)-+$ indiquée ci-dessus en a), et donnent l'ordre de grandeur de la partie - $(+)$ (ombre et pénombre proche).

Le succès de cette méthode est en fait assez surprenant. En effet, s'il se confirme que l'interaction ne fait intervenir aucun effet collectif (autre que les équations de Maxwell), cela suggère que la longueur d'interaction $\lambda_{c}$ dans le vent solaire est plus grande que le rayon lunaire, c'est-à-dire $1700 \mathrm{~km}$. On obtiendrait donc pour $\lambda_{c}$ les inégalités :

$$
1700 \mathrm{~km}=R_{!}<\lambda_{c}<10 R_{\oplus}=63700 \mathrm{~km} .
$$

Il y a là une propriété essentielle du vent solaire, qui, si elle est confirmée, pourra aider à déterminer théoriquement $\lambda_{c}$.

Conclusion. - Cet exposé a plus passé en revue les résultats acquis que posé les problèmes actuels. Il est certain qu'une quantité considérable d'informations ont été accumulées depuis 7 ans sur l'environnement magnétique au voisinage de l'orbite terrestre.

Les grandes observations exploratoires devront avoir désormais pour objectif d'atteindre l'orbite de Jupiter, tant pour étudier la variation radiale du vent solaire que la magnétosphère de cette planète, et d'autre part il serait intéressant d'étudier la structure du champ magnétique hors du plan de l'écliptique. Il reste également à rechercher les modalités de l'interaction du vent solaire avec toutes les planètes. Enfin l'étude des discontinuités du vent solaire, la propagation d'ondes dans le vent ouvrent un vaste domaine à l'expérimentation.

Pourtant les problèmes les plus difficiles posés actuellement sont peut-être relatifs à la compréhension profonde des phénomènes que nous avons exposés ici. Le problème fondamental de l'origine du comportement fluide du vent solaire n'est pas résolu. Le mécanisme d'interaction du vent avec la queue de la magnétosphère n'est pas élucidé. D'une façon générale, l'activité géomagnétique est très probablement contrôlée par le vent solaire, plus spécialement par le champ magnétique du vent solaire. Mais on ignore encore comment. C'est pourquoi les mesures magnétiques externes constitueront longtemps encore un domaine de recherche extrêmement fécond.

Pour conclure, revenons aux trois objets intrinsèque- 
ment magnétiques du système solaire : le Soleil, la Terre, Jupiter. Apparemment, nous ne sommes pas encore près d'entrer dans l'ère de l'étude expérimentale des structures internes. C'est pourquoi, rien ou presque rien n'étant connu dans ce domaine, nous l'avons délibérément écarté au cours de l'exposé; il n'en réunit pas moins trois des plus préoccupantes énigmes actuelles qu'offre le système solaire.

\section{BIBLIOGRAPHIE}

[1] WARWICK (J. W.), Radiophysics of Jupiter, Space Sci. Rev., 1967, 6, 841-891.

[2] BABCOCK (H. W.) et BABCOCK (H. D.), The sun's magnetic field, Astrophys. J., 1955, 121, 349-366.

[3] Behannon (K. W.), Intrinsic magnetic properties of the lunar body, J. Geophys-res., 1968, 73, 7257-7268.

[4] VAN AliLEN (J. A.), KRimigis (S. M.), Franck (L. A.) et ARMSTRONG (J. P.), Observed absence of energetic protons and electrons near Venus, $J$. Geophys-res., 1968, 73, 421-425.

[5] Smith (E. J.), Davis (L.), Coleman (P. J.) et JoNES (D. E.), Magnetic field measurements near Mars, Science, 1965, 149, 1241-1242.

[6] RoberTs (J. A.), Radio emission from the planets, Planet Space Sci., 1963, 11, 221-259.

[7] PArker (E. N.), Dynamical theory of the solar wind, Space Sci. Rev., 1965, 4, 666-708.

[8] AXFord (W. I.), Dessleer (A. J.) et GotTlimeB (B.), Termination of solar wind and solar magnetic field, Astrophys. J., 1963, 137, 1268-1278.

[9] Hundhausen (A. J.), Direct observation of solar wind particles, Space Sci. Rev., 1968, 8, 690-749.

[10] NEss (N. F.) et Wricox (J. M.), Extension of the photospheric magnetic field into interplanetary space, NASA X, 1965, 612, 65-79.

[11] SPITZER (L.), Physics of fully ionized gases, Interscience, 1956, chap. 2.

[12] Coleman (P. J.), Variations in the interplanetary magnetic field, Mariner II, J. Geophys-res., 1966, 71, 5509-5531.

[13] Wricox (J. M.) et NEss (N. F.), Quasistationary corotating structures in the interplanetary medium, J. Geophys-res., 1965, 72, 5793-5805.

[14] Coleman (P. J.), Davis (L.), Smith (E. J.) et JonES (D. E.), The polarity pattern of the interplanetary magnetic field during solar rotations 1798-1808, J. Geophys-res., 1967, 72, 16371643.

[15] Ness (N. F.), Scearce (C. S.) et Cantarano (S.), Preliminary results from the Pioneer VI magnetic field experiment, J. Geophys-res., 1966, 71, 33053313.

[16] NEss (N. F.), Simultaneous measurements of the interplanetary magnetic field, J. Geophys-res., 1966, 71, 3319-3323.

[17] CaIn (J. C.), Models of the earth's magnetic field, in Radiation trapped in the earth's magnetic field, D. Reidel, Dordrecht, 1966.

[18] Bernard (J.), Kosik (J. C.), Lavai (G.), Peilat (R.) et PHILIPPON (J. P.), Représentation optimale du potentiel géomagnétique dans le repère d'un dipôle décentré, incliné, C.E.R.T.S. NT 01-09, Toulouse, 1968.
[19] Crifo (J.-F.), Dynamique des ceintures de radiations, partie 1, L.P.S.P. E-4, 1968.

[20] Price (A. T.), Daily variations of the geomagnetic field, Space Sci. Rev., 1969, 9, 151-197.

[21] MEAD (G. D.) et BEARD (D. B.), Shape of the geomagnetic field solar wind boundary, J. Geophysres., 1964, 69, 1169-1179.

[22] Midgley (J. E.) et Davis (L.), J. Geophys-res., 1963, 68, 5111-5123.

[23] Spreiter (J. R.), Summers (A. L.) et ALSkne (A. Y.), Hydromagnetic flow around the magnetosphere, Planet Space Sci., 1966, 14, 223-253.

[24] Spreiter (J. R.), Alskne (A. Y.) et Summers (A. L.), External aerodynamics of the magnetosphere, in Physics of the magnetosphere, D. Reidel, 1968.

[25] FAIRFIELD (D. H.), The ordered magnetic field of the magnetosheath, J. Geophys-res., 1967, 72, 5865-5877.

[26] MEAD (G. D.), Deformation of the geomagnetic field by the solar wind, J. Geophys-res., 1964, 69, 1181-1195.

[27] Midgley (J. E.), Perturbation of the geomagnetic field A spherical harmonic expansion, J. Geophysres., 1964, 69, 1197-1200.

[28] FAIRFIELD (D. H.), Average magnetic field configuration of the outer magnetosphere, J. Geophysres., 1968, 73, 7329-7338.

[29] Mrhalov (J. D.) et Soneit (C. P.), The cislunar geomagnetic tail gradient in 1967, J. Geophys-res., 1968, 73, 6837-6841.

[30] NESS (N. F.), Probable observation of the geomagnetic tail at $10^{3} \mathrm{R}_{\oplus}$ by Pioneer 7, NASA REPT X, 1967, 612, 67-183.

[31] Hones Jr (E. W.), Review and interpretation of particle measurements made by the Vela satellites in the magnetotail, in Physics of the magnetosphere, D. Reidel, 1968.

[32] Ness (N. F.), Behannon (K. W.), Scearce (C. S.) et CANTARANo (S. C.), Early results from the magnetic field experiment on lunar Explorer XXXV, J. Geophys-res., 1967, 72, 5769-5778.

[33] BeHANNON (K. W.), Intrinsic magnetic properties of the lunar body, J. Geophys-res., 1968, 73, 72577268.

[34] Ness (N. F.), Behannon (K. W.), Taylor (H. E.) et Whang (Y. C.), Perturbations of the interplanetary magnetic field by the lunar wake, J. Geophys-res., 1968, 73, 3421-3440.

[35] TAylor (H. E.), Behannon (K. W.) et Ness (N. F.), Measurements of the perturbed-interplanetary magnetic field in the lunar wake, $J$. Geophys-res., 1968, 73, 6723-6735.

[36] Jonhson (F. S.) et Midgley (J. E.), Notes on the lunar magnetosphere, J. Geophys-res., 1968, 73, 1523-1532. 